\title{
The Role of Attention in the Maintenance of Feature Bindings in Visual Short-Term Memory
}

\author{
Jeffrey S. Johnson and Andrew Hollingworth \\ University of Iowa
}

\author{
Steven J. Luck \\ University of California, Davis
}

\begin{abstract}
This study examined the role of attention in maintaining feature bindings in visual short-term memory. In a change-detection paradigm, participants attempted to detect changes in the colors and orientations of multiple objects; the changes consisted of new feature values in a feature-memory condition and changes in how existing feature values were combined in a binding-memory condition. In the critical experiment, a demanding visual search task requiring sequential shifts of spatial attention was interposed during the delay interval of the change-detection task. If attention is more important for the maintenance of feature bindings than for the maintenance of unbound feature values, the attention-requiring search task should specifically disrupt performance in the binding-memory task. Contrary to this proposal, it was found that memory for bindings and memory for features were equally impaired by the search task.
\end{abstract}

Keywords: feature binding, visual short-term memory, attention

Converging evidence from electrophysiological recording studies in monkeys and functional imaging studies in humans has suggested that object properties - such as color, form, size, and direction of motion-are encoded in a distributed manner across different neural populations in the visual cortex (Tootell, Dale, Sereno, \& Malach, 1998). This type of coding in the visual system leads to the problem of linking the different features of an object together so that features arising from different objects are not perceived as arising from the same object, which is called the binding problem (for reviews, see Luck \& Beach, 1998; Roskies, 1999; Treisman, 1996). According to Treisman's (1993; Treisman \& Gelade, 1980) highly influential feature integration theory, this problem is solved by the sequential allocation of visual selective attention to regions of space, which allows the features of a given object to be linked through their shared spatial location. Once the features of an object are linked, attention plays a role in the construction of a limited number of relatively complex object representations (Kahneman, Treisman, \& Gibbs, 1992). Such object representations make it possible to maintain the experience of a unified object across changes in position or physical properties through time. Given the central role of attention in constructing bound object representations at the level of perception, it is natural to ask whether maintaining bound representations of features in

Jeffrey S. Johnson and Andrew Hollingworth, Department of Psychology, University of Iowa; Steven J. Luck, Center for Mind and Brain and Department of Psychology, University of California, Davis.

This research was made possible by National Institute of Health Grants R01 MH63001 and R01 MH65034 awarded to Steven J. Luck. Portions of this research were presented at the 45th Annual Meeting of the Psychonomic Society, Minneapolis, Minnesota, November 2004, and at the Fifth Annual Meeting of the Vision Sciences Society, Sarasota, Florida, May 2005.

Correspondence concerning this article should be addressed to Jeffrey S. Johnson, Department of Psychology, University of Iowa, 11 Seashore Hall E, Iowa City, IA 52242-1407. E-mail: jeffrey-johnson-2@uiowa.edu visual memory requires more attention than maintaining simple features in visual memory.

Evidence suggesting an important role for attention in the maintenance of bound object representations has come from studies investigating the role of visual memory in scene representation (Rensink, 2000). A commonly used method in this area is the change-detection task, in which observers view complex images and look for changes that occur during a visual disruption (such as a saccade or brief interstimulus interval). Observers often fail to notice highly salient changes in visual scenes that occur outside of the current focus of attention, a phenomenon called change blindness (Rensink, 2000; Simons \& Levin, 1997). To explain this phenomenon, visual memory for objects has been proposed to be either nonexistent (O'Regan, 1992; O'Regan \& Nöe, 2001) or limited to the currently attended object (Rensink, 2000, 2002), with little to no accumulation of information from previously attended objects. According to the latter proposal, once attention is withdrawn from an object, feature bindings come undone, and the representation of an object in visual memory disintegrates into its constituent features, making it impossible to detect changes to this object. Wolfe has made a similar proposal in the context of visual search (Horowitz \& Wolfe, 1998; Wolfe, 1999), claiming that only the currently attended object during search is maintained in a bound format.

In keeping with these proposals, Wheeler and Treisman (2002) studied the binding of features in visual short-term memory (VSTM) and concluded that the maintenance of feature bindings in VSTM depends on sustained attention (Rensink, 2000). To investigate this issue, they used a variant of the change-detection task described above (see Figure 1A), in which observers view a brief sample array containing one or more objects that they must try to remember. After a brief retention interval, a test array is presented, and the observers compare the test array with the sample array. In most experiments, the test array and sample array are identical on $50 \%$ of trials and differ in some subtle way on the remaining trials, and the observers simply make an unspeeded same/different re- 


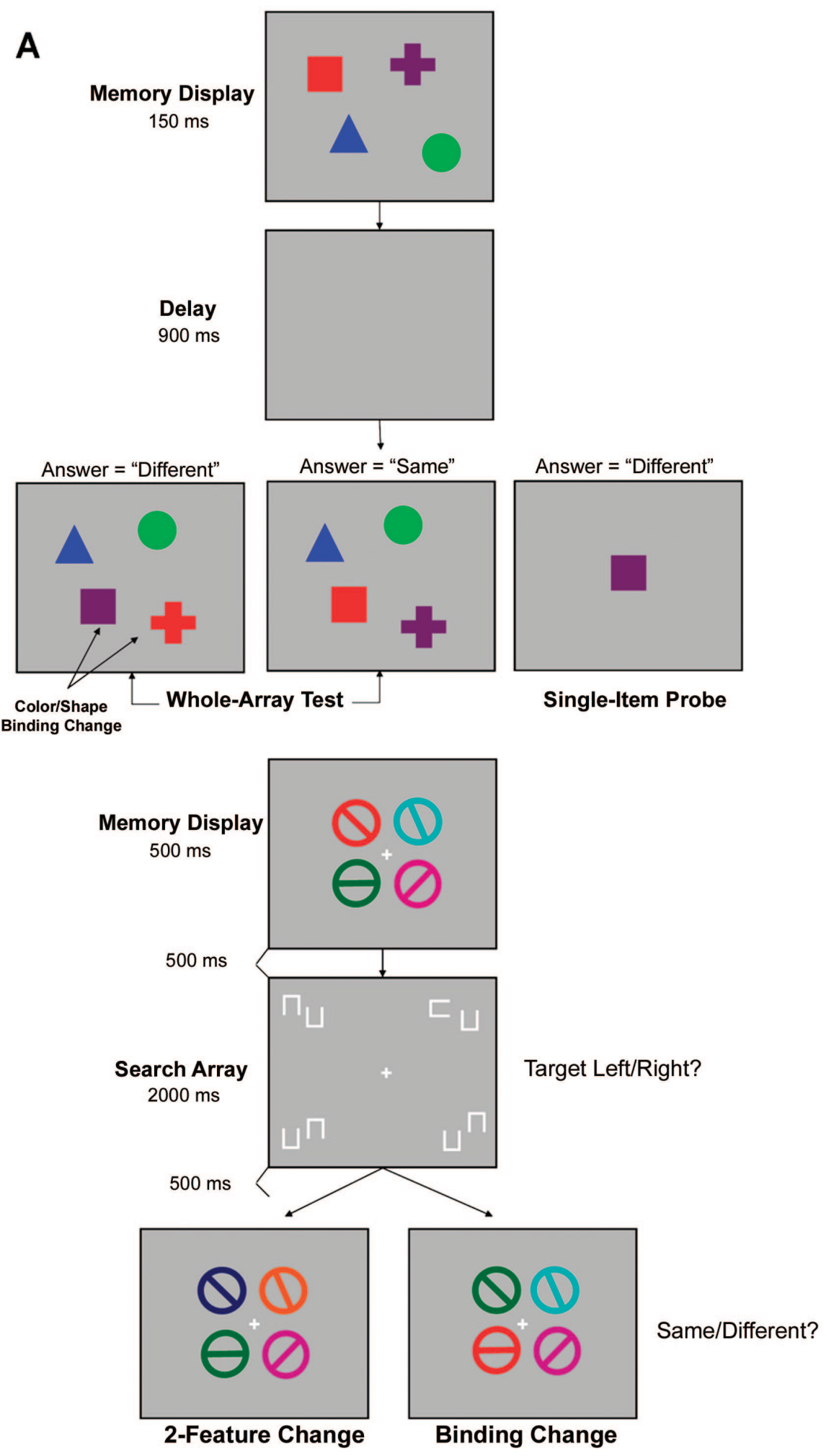


sponse to indicate whether they noticed a difference (see, e.g., Luck \& Vogel, 1997; Phillips, 1974).

To examine feature binding in VSTM, Wheeler and Treisman (2002) included an explicit binding-memory condition in the standard change-detection paradigm. In this condition, all of the same features were presented again at test, but the particular pairing of features was changed. As shown in Figure 1A, for instance, the initial memory display might contain a red square, a blue triangle, a green star, and a violet plus sign. On a binding-trial, all four shapes and all four colors were presented at test, but color was swapped between two shapes (e.g., a red plus sign and a violet square). Successful change detection in the binding condition required participants to bind each of the sample colors to a specific shape and to retain these bindings throughout the delay interval. ${ }^{1}$ The binding condition was compared with a 2-feature condition (termed the either condition in Wheeler \& Treisman, 2002), in which participants monitored for a change in either of the two feature dimensions (color or shape). To equate the magnitude of the change in the 2-feature condition with that in the binding condition, two objects changed to new values along one feature dimension on change trials (e.g., the colors of two objects changed to new colors not present in the memory display, such as a pink plus sign and an orange square). The 2-feature condition required memory for both feature dimensions but did not require memory for the binding of the features.

It was found that accuracy in the binding condition was generally worse than that in the 2 -feature condition. Mean accuracy was $84 \%$ for color change detection in the 2 -feature condition, $74 \%$ for shape change detection in the 2-feature condition, and $70 \%$ for binding change detection. A similar binding deficit was found when participants were required to bind color and position information. Importantly, binding-specific decrements appeared only when all memory display items were presented at test (see Figure 1A), with no binding decrement when the test array only contained a single probe item.

Wheeler and Treisman (2002) hypothesized that the bindingspecific impairments in the whole-array test conditions may have occurred as a result of attentional distraction. In particular, they proposed that attention is required to actively maintain bound object representations in working memory, just as attention is required to bind features in visual perception. When a new display

Figure 1 (opposite). A: Example of the type of stimuli used in Experiments 4A and 4B of Wheeler and Treisman (2002). In the whole-array test condition, the position of each of the items was changed at test; the color-shape pairings remained identical on no-change trials, whereas the pairing of color and shape for two of the items was changed at test on change trials. In the single-item probe condition, a single item was presented at the center of the screen at test, and this item was either identical to one of the items from the sample array or combined the color of one sample item with the shape of a different sample item. B: Example of the stimuli used in Experiment 1 of the present study. The locations of the items were not changed between the sample and test arrays. The sample and test arrays were identical on no-change trials. For change trials in the 2-feature condition, the colors or the orientations of two of the memory display items were changed to new values at test. For change trials in the binding condition, the color-orientation pairing of two items was changed at test. Note that the items are not drawn to scale. is presented at test, attentional resources that were being used to maintain the bindings in memory are withdrawn to process the objects in the test display, resulting in a loss of bound representations of the original items and a consequent decline in changedetection performance. In contrast, when a single item is presented at test, fewer attentional resources are required to process the test display, and change-detection performance therefore remains efficient. On the basis of this reasoning, Wheeler and Treisman concluded that the maintenance of bindings in VSTM depends on sustained attention; we call this the attention maintenance hypothesis.

Contrary to this proposal, Hollingworth and Henderson (2002) have suggested that a limited number of higher level visual object representations can be retained briefly in VSTM following the withdrawal of attention, supporting the creation of longer term scene representations (see also Irwin, 1992; Irwin \& Zelinsky, 2002). This proposal was supported by the results of experiments examining the detection of visual changes to objects in real-world scenes. In these experiments, change-detection performance remained highly accurate even when many fixations on other objects intervened between target fixation and test (Hollingworth, 2004; Hollingworth, Williams, \& Henderson, 2001). Because visual attention is tightly coupled to movements of the eyes (Hoffman \& Subramaniam, 1995), these data suggest that object representations remain intact in visual memory in the absence of sustained attention.

A similar proposal had been made by Luck and Vogel (1997; Vogel, Woodman, \& Luck, 2001) to explain the results of a series of change-detection experiments investigating the storage of features and objects in VSTM. In these experiments, it was found that participants were just as good at remembering multifeature objects as individual features (but, for important qualifications of these results, see Olson \& Jiang, 2002; Wheeler \& Treisman, 2002; Xu, 2002, 2004). On the basis of these findings, Luck and Vogel proposed the integrated object hypothesis, which holds that individual features are bound into object representations by perceptual processes and that these representations remain integrated in VSTM without requiring any resources beyond those required to maintain individual features.

The results reported by Wheeler and Treisman (2002) do not really challenge this hypothesis, because Wheeler and Treisman never directly manipulated the availability of attention during the maintenance interval. Although it is possible that presenting the full array at test led to attentional distraction, there is no compelling reason to think this was so, especially given that previous studies have found that change detection is improved when the full array is presented at test compared with when a single test object is displayed (Jiang, Olson, \& Chun, 2000). Additionally, the conclusion of Wheeler and Treisman was only weakly supported by

\footnotetext{
${ }^{1}$ At present, the change-detection task described here, which was also used in the present series of experiments, is the most widely accepted method for examining feature binding in VSTM. However, it is possible that performance in the binding conditions used here may reflect other factors in addition to feature binding in VSTM (see, e.g., Saiki \& Miyatsuji, in press). Thus, in the future it will be important to seek converging support for these findings using different measures of feature binding in VSTM.
} 
their data. Although clear decrements were found for binding memory with whole arrays at test, performance remained quite good overall, with $79 \%$ correct in the whole-array color-positionbinding condition of Experiment $3 \mathrm{~A}$ and $70 \%$ correct in the whole-array color-shape-binding condition of Experiment 4A. One would expect much more profound impairments had the bound object representations needed to detect changes simply fallen apart with the withdrawal of attention, as Wheeler and Treisman proposed.

There are further methodological and statistical issues that make the Wheeler and Treisman (2002) data difficult to interpret. The binding deficit was observed in Experiments $3 \mathrm{~A}$ and $4 \mathrm{~A}$ of that study (whole-array tests). In Experiment 3A, memory was tested for bindings of color and location. In the 2-feature condition, participants monitored for either a change in color or a change in location. When color changed, two colors were replaced by new colors not present in the initial display. When location changed, two objects were moved to previously unoccupied locations. In the binding condition, participants monitored for a change in colorposition binding. When binding changed, two colors switched locations. As described above, accuracy was higher in the 2-feature conditions than in the binding condition, which Wheeler and Treisman interpreted as a binding deficit caused by attentional distraction from the presentation of the whole array at test.

The problem with this design is that when location changed in the 2-feature condition, the overall spatial configuration changed as well (because two colors were moved to new locations). However, changes in the binding condition did not alter the spatial configuration of objects (because colors traded locations). Thus, the feature/binding manipulation was confounded with a change in configuration. It is well established that changes in configuration are highly salient (Jiang et al., 2000; Simons, 1996). Configural encoding of the array would explain the high levels of accuracy observed when position changed in the 2-feature condition and the lack of a set size effect in that condition (as suggested by Wheeler $\&$ Treisman, 2002). Finally, configural encoding would account for the absence of a binding deficit when a single item was presented at test (Wheeler \& Treisman, 2002): Because only a single test item was present (disrupting the configuration in all conditions), configural encoding could no longer benefit the 2 -feature condition.

Given these considerations, the apparent binding deficit in Experiment 3A of Wheeler and Treisman (2002) cannot be confidently attributed to a difference in memory for features versus memory for bindings. More generally, color-position binding is not an ideal means to examine binding in VSTM, because spatial information and object information are stored in partially independent systems (Baddeley \& Logie, 1999; Courtney, Ungerleider, Keil, \& Haxby, 1996). Binding across these subsystems may follow different rules than binding within a subsystem. Consequently, the critical issue is whether binding within the object subsystem requires sustained attention, and that question is better answered using visual features that are known to depend directly on the object VSTM subsystem.

The empirical support for a binding deficit in Wheeler and Treisman (2002) therefore rests on their Experiment 4A. In this experiment, the binding of shape and color was examined, as described above (see Figure 1A). The method used visual features that are known to engage VSTM and was designed to rule out contributions from spatial memory. To conclude that a binding deficit exists in these experiments, performance in the binding condition must be directly compared with performance in the 2 -feature condition. However, in the absence of an explicit computational model of binding in VSTM, it is not clear whether the appropriate comparison is between the binding condition and the more difficult of the two features in the 2-feature condition (because binding performance will be limited by memory for each feature) or between the binding condition and the average of the two features in the 2-feature condition. In Wheeler and Treisman's Experiment 4A, participants were correct $70 \%$ of the time in the binding condition, compared with $74 \%$ of the time for the more difficult of the two features (i.e., shape) and $79 \%$ of the time when color and shape are averaged together. Although both contrasts suggest numerical decrements in binding relative to feature memory, Wheeler and Treisman did not report whether these relatively small effects were statistically reliable; no direct statistical comparison of the 2-feature and binding conditions was reported. ${ }^{2}$ Thus, the results from Wheeler and Treisman's Experiment 4A do not provide very strong evidence of a binding deficit. Furthermore, because attention was not directly manipulated in their experiments, any binding deficits that may have existed cannot be confidently attributed to the withdrawal of attention from the items in VSTM.

Although the Wheeler and Treisman (2002) experiments do not provide a strong test of the hypothesis that sustained attention is necessary to maintain bindings in VSTM, that hypothesis can be directly tested by introducing an attentionally demanding task during the maintenance interval of a change-detection task requiring memory for visual features and memory for the binding of features. If attention is necessary for the maintenance of feature bindings, binding memory should be substantially impaired compared with feature memory when attention is withdrawn to perform the secondary task.

Several experiments have attempted such a manipulation, but methodological and statistical issues have made it difficult to draw firm conclusions from these studies. For example, Stefurak and Boynton (1986) reported evidence consistent with the attention maintenance hypothesis in a series of experiments comparing memory for features with memory for bindings when a mental arithmetic task was completed concurrently with the memory task. However, their experiments required participants to remember arbitrary associations between unique colors and real-world shapes (e.g., a giraffe, a penguin, an elephant) that are already strongly related to particular colors (e.g., giraffe and yellow) and are incompatible with other color pairings (e.g., giraffe and red). As a result, the binding decrements in their experiments may have arisen as a result of interference from previous color-shape associations stored in long-term memory rather than as a result of the

\footnotetext{
${ }^{2}$ The statistical evidence used to support the conclusion of a binding deficit in Experiment 4A of Wheeler and Treisman (2002) was a reliable analysis of variance over four conditions: color only (in which participants monitored only for color changes), shape only (in which participants monitored only for shape changes), 2-feature (color or shape), and binding. Accuracy in the color-only and shape-only conditions was significantly higher than accuracy in the other conditions, so the source of the effect cannot have been a binding deficit. No reported analyses compared accuracy in the 2-feature condition with accuracy in the binding condition.
} 
attentional demands of the mental arithmetic task (Allen, Baddeley, \& Hitch, 2006).

Another series of experiments that aimed to test the attention maintenance hypothesis was reported by Yeh, Yang, and Chiu (2005). Participants in this study completed a change-detection task similar to the color-location memory task of Wheeler and Treisman (2002) but with the inclusion of a speeded letter recognition task during the delay interval. However, Yeh et al.'s analysis only considered the hit rate on change trials, despite the fact that there were quite large differences in false alarm rate on the same trials, making it difficult to draw firm conclusions on the basis of their results.

In the present study, we tested the attention maintenance hypothesis in a series of change-detection experiments in which participants remembered simple objects varying in color and orientation or color and shape. In the critical experiment, binding memory was compared with feature memory when a difficult visual search task was completed during the delay interval. The attention task used here has been shown to require sequential shifts of attention to find the target (Woodman \& Luck, 2003). Contrary to the attention maintenance hypothesis, and contrary to visual transience theories of visual memory more generally, we found that binding memory and feature memory were equally impaired by the search task.

\section{Experiment 1}

In our first experiment, we provided a stringent test of the attention maintenance hypothesis by interposing a demanding visual search task during the delay interval of a change-detection task. The sequence of events in a trial and examples of the stimuli used are illustrated in Figure 1B.

The sample and test objects in this experiment were colored, oriented bars surrounded by a circle. The enclosing circles were task irrelevant and were intended to minimize the likelihood that participants encoded the global configuration formed by the oriented bars rather than the individual orientations (Delvenne, Braithwaite, Riddoch, \& Humphreys, 2002). Visual search stimuli were small square outlines with a gap opening to the left, right, up, or down. Because previous work (Luck \& Vogel, 1997) had demonstrated that memory for two or more features that were part of the same object was just as efficient as memory for single features, participants completed only two memory conditions in this experiment: (a) a 2-feature memory condition, in which either the color or the orientation of two of the items could be changed to new values at test, and (b) a binding-memory condition, in which either the color or the orientation of two of the items could be swapped at test (see Figure 1B). Each participant completed these two memory conditions both with and without a concurrent search task. Under the strong hypothesis that sustained attention is necessary to maintain feature bindings, but not the features themselves, performance in the binding condition should be near chance when the visual search task is performed during the delay interval, whereas performance in the 2-feature memory condition should be unaffected by the visual search task. That is, a significant binding deficit should be observed.

One methodological difference between this experiment and the whole-array test experiments of Wheeler and Treisman (2002) concerned the positions of the objects in the test array. In their color-shape binding experiment, Wheeler and Treisman attempted to render position information completely uninformative by changing the locations of all the items between the presentation of the memory display and the test display (see Figure 1A). Although this certainly makes it impossible to use location information to complete the task, we reasoned that it might also increase the complexity of the comparison process at test by requiring participants to make a larger number of overall comparisons to detect a change. That is, when location is held constant at test, each item in the test display need only be compared with the one item at the corresponding location in memory to detect a change to that item. In contrast, when the locations are scrambled at test, it is not clear which item in memory should be compared with which item in the test array, which may overload the comparison process. Because we were interested in assessing the storage of information in VSTM rather than the process of comparing VSTM representations with sensory inputs, we did not adopt this approach. Instead, we simply presented each of the test items at the same locations they had occupied in the initial display.

\section{Method}

Participants. Fifty University of Iowa undergraduate volunteers (18 men and 32 women) participated in this experiment. A set size of three items was used with half of the participants, and a set size of four items was used with the other half. Participants received class credit or monetary compensation for their participation. All participants reported normal or corrected-to-normal visual acuity and normal color vision. Five participants were replaced because they failed to perform significantly better than chance (as assessed with a binomial test) when the binding and 2-feature memory tasks were performed without the visual search task.

Stimuli. Stimulus presentation and response recording was controlled by a Macintosh G4 computer running either PsyScript software (Bates \& D'Oliveiro, 2003) or Matlab 5.2.0 using the Psychophysics Toolbox extensions (Experiment 3; Brainard, 1997; Pelli, 1997). Stimuli were presented against a gray background $\left(6.15 \mathrm{~cd} / \mathrm{m}^{2}\right)$ on a $17-$ in. $(43.18-\mathrm{cm}) \mathrm{CRT}$ computer monitor at a viewing distance of approximately $57 \mathrm{~cm}$. Memory stimuli consisted of small, colored circles $\left(1.74^{\circ}\right.$ in diameter), each with an identically colored oriented line segment spanning its interior (see Figure 1B). Both the circles and the bars had a thickness of $0.23^{\circ}$. Responses were collected using a handheld game pad.

Sample arrays consisted of either three or four items presented at each of the corners of an imaginary $2.27^{\circ} \times 2.27^{\circ}$ square centered at fixation. Colors were created using the FreeHand MX HLS color picker (Adobe Systems, San Jose, CA), with lightness set to $50 \%$ and saturation set to $100 \%$. Twelve different hues were chosen at $30^{\circ}$ intervals around the color circle, starting at $0^{\circ}$. The $x, y$, and luminance values for each resulting color were measured with a Tektronix model J17 colorimeter (Richardson, TX), using the $1931 \mathrm{CIE}$ color coordinate system, and were as follows: red $\left(x=.66, y=.32 ; 15.49 \mathrm{~cd} / \mathrm{m}^{2}\right)$, orange $(x=.59, y=.37 ; 23.2$ $\left.\mathrm{cd} / \mathrm{m}^{2}\right)$, yellow $\left(x=.45, y=.47 ; 57.62 \mathrm{~cd} / \mathrm{m}^{2}\right)$, lime-green $(x=$ $\left..35, y=.54 ; 45.14 \mathrm{~cd} / \mathrm{m}^{2}\right)$, green $\left(x=.35, y=.57 ; 42.16 \mathrm{~cd} / \mathrm{m}^{2}\right)$, aqua $\left(x=.28, y=.47 ; 43.07 \mathrm{~cd} / \mathrm{m}^{2}\right)$, cyan $(x=.22, y=.29 ; 46.88$ $\left.\mathrm{cd} / \mathrm{m}^{2}\right)$, cyan-blue $\left(x=.17, y=.12 ; 12.92 \mathrm{~cd} / \mathrm{m}^{2}\right)$, blue $(x=.15$, $\left.y=.06 ; 5.48 \mathrm{~cd} / \mathrm{m}^{2}\right)$, purple $\left(x=.20, y=.09 ; 8.73 \mathrm{~cd} / \mathrm{m}^{2}\right)$, 
magenta $\left(x=.33, y=.15 ; 20.8 \mathrm{~cd} / \mathrm{m}^{2}\right)$, and violet-red $(x=.53$, $\left.y=.25 ; 16.35 \mathrm{~cd} / \mathrm{m}^{2}\right)$.

To avoid large variations in the magnitudes of color changes, we placed individual colors into four different groups, each of which contained three different colors separated by $120^{\circ}$ in color space. For example, Group 1 contained the colors red, green, and blue, whereas Group 2 contained orange, aqua, and purple. The colors presented on a given trial were selected at random from within two of these groups: for example, two from one group (e.g., red and green from Group 1) and two from another group (e.g., aqua and purple from Group 2), with a set size of four. When a color change occurred at test, two of the memory stimuli drawn from different groups (e.g., red and aqua) were changed to the remaining unused values in their group. Thus, in the present example, the red item would change to blue and the aqua item would change to orange. In contrast, for binding-memory trials, the two items with colors drawn from the same group (e.g., the red item and the green item) switched their colors at test. Thus, for both trial types, when an item's color changed at test, it was always to another item that was exactly $120^{\circ}$ away in color space.

In a similar fashion, 12 different orientations were selected at $15^{\circ}$ intervals beginning at $0^{\circ}$ (i.e., horizontal) and ending at $165^{\circ}$. Individual orientations were then sorted into four different groups of three items, chosen at $60^{\circ}$ intervals. Thus, Group 1 contained $0^{\circ}$, $60^{\circ}$ and $120^{\circ}$ orientations; Group 2 contained $15^{\circ}, 75^{\circ}$, and $135^{\circ}$ orientations; and so forth. The orientations used on a given trial were selected exactly as they were for colors: two from one group and the remaining item(s) from another group. Similarly, when an orientation change occurred at test, one of the items from each group always changed to the remaining item in that group. This ensured that the orientation change magnitude was never less than $60^{\circ}$, although it could be as high as $120^{\circ}$ depending on the specific memory items selected on a given trial.

The search arrays consisted of eight light-gray $\left(15.06 \mathrm{~cd} / \mathrm{m}^{2}\right)$ items, one of which was the target (see Figure 1B). The nontargets were $0.53^{\circ} \times 0.53^{\circ}$ outlined squares with one side open on the top or bottom. The target was a square that was identical except that the open side faced to the left or the right. A total of eight search items were presented, with two presented at random locations within each of four $2.27^{\circ} \times 2.27^{\circ}$ regions centered $5.5^{\circ}$ diagonally from the fixation cross at the center of the screen, with a minimum center-to-center separation of $1^{\circ}$.

Procedure. Participants completed single-task trials and dualtask trials in separate blocks. Dual-task trials began with the appearance of a fixation cross at the center of the screen for 500 $\mathrm{ms}$, followed by a $500-\mathrm{ms}$ presentation of the memory display. This was followed by a 500-ms delay interval and then a 2,000-ms presentation of the visual search array. Participants were instructed to make a speeded response to the search array, indicating whether the target square opened to the left or the right by pressing one of two different buttons on a handheld game pad, with a left-hand response for a leftward opening and a right-hand response for a rightward opening. The offset of the search array was followed by another 500-ms blank interval and the appearance of a test display. Each item in the test display appeared at the same location it had occupied in the initial memory display. When the test display appeared, participants made an unspeeded same or different response using a handheld game pad, with a left-hand response for same and a right-hand response for different. Changes occurred on
$50 \%$ of trials, with color and orientation changes being equally likely. Participants were instructed to strive for accuracy rather than speed, and they were given feedback in the form of a highpitched tone when they made an incorrect response. Single-task trials were identical except that for memory-only trials the search arrays never contained a target, and for search-only trials no change ever occurred. Participants were instructed to simply ignore the irrelevant task for these trials, and no response was required.

Participants completed a total of five trial blocks in this experiment, performing one block of the search-only condition and four blocks in which the 2-feature memory and binding-memory tasks were performed with and without the visual search task. For half of the participants, the memory displays contained three items, whereas for the other half, the memory displays contained four items. Two set sizes were included in this experiment to provide a range of accuracy levels, which is important to rule out floor effects. That is, if accuracy in both the binding and 2-feature memory conditions is found to be near the floor, it may be difficult to see a small, but nonetheless real, binding-specific deficit when the search task is performed during the delay interval.

At the beginning of the experimental session, participants read printed instructions describing each task and the nature of the change they were to look for in different blocks. The order of trial blocks was counterbalanced using a Latin square. Each trial block contained 48 trials, for a total of 240 trials. Participants received $12-16$ practice trials at the beginning of each block.

To rule out contributions from verbal working memory, we had participants perform a concurrent articulatory suppression task. Specifically, three randomly selected digits were presented on the video monitor at the beginning of each trial block, and participants were instructed to repeat these numbers aloud at a steady rate throughout each trial. Previous research has shown that this task effectively discourages participants from recoding and storing visual information verbally (Baddeley, 1986; Besner, Davies, \& Daniels, 1981; Murray, 1968).

\section{Results and Discussion}

An alpha level of .05 was used as the criterion for statistical significance in all experiments, and the signal detection sensitivity measure $d^{\prime}$ was the primary dependent measure. We also conducted analyses on log-transformed percentage correct for each experiment. Log-transformed percentage correct produced the same pattern of statistical significance as $d^{\prime}$ across all experiments. Proportional hits and false alarms for each of the experiments reported here are provided in the Appendix and can be used to compute other measures of performance, such as the Cowan/ Pashler $K$ (Cowan, 2001; Pashler, 1988).

Visual search. Figure 2A reveals that accuracy was uniformly high in the search task, with greater than $95 \%$ correct performance in all conditions and across both memory set sizes. Confirming this, a repeated measures analysis of variance (ANOVA) with search condition (alone, with binding memory, with 2-feature memory) as a within-subject factor and memory set size (three or four items) as a between-subjects factor, yielded no significant main effects or interactions (all $p \mathrm{~s}>.15$ ).

In contrast, mean reaction time (RT) was shorter when the search task was performed by itself ( $\mathrm{RT}=1,109 \mathrm{~ms}$ ) than when it 


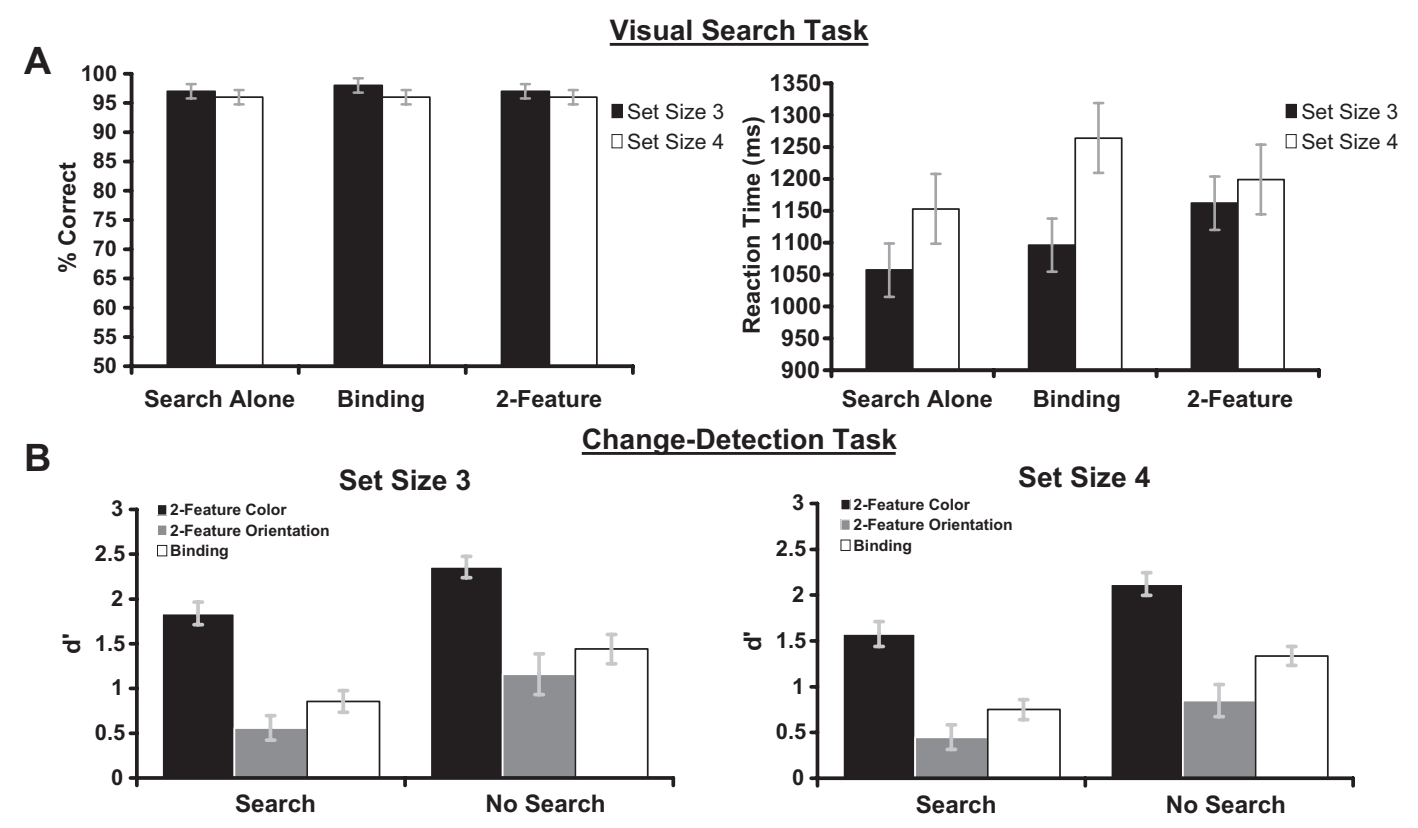

Figure 2. Results from the visual search (A) and memory (B) tasks from Experiment 1. Error bars reflect $95 \%$ confidence intervals, as described by Loftus and Masson (1994).

was completed concurrently with either the binding memory task $(\mathrm{RT}=1,177 \mathrm{~ms})$ or the 2-feature memory task $(\mathrm{RT}=1,180 \mathrm{~ms})$. Additionally, the search task was performed faster with a memory set size of three items (RT $=1,105 \mathrm{~ms}$ ) than with a memory set size of four items ( $\mathrm{RT}=1,206 \mathrm{~ms}$ ). In keeping with these data, a repeated measures ANOVA revealed a significant main effect of search condition on RT, $F(2,96)=5.66, p=.005$, and a significant Search Condition $\times$ Memory Set Size interaction, $F(2$, $96)=3.38, p=.04$. The main effect of memory set size approached but did not quite reach significance, $F(1,48)=3.43, p=$ .07. Of central importance, search was no slower in the binding condition than in the 2 -feature condition $(F<1)$.

Change detection. The memory results are summarized in Figure 2B. Overall, accuracy was worse in the binding-memory task $\left(d^{\prime}=1.09\right)$ than in the 2 -feature memory task $\left(d^{\prime}=1.33\right)$. Additionally, concurrent performance of the visual search task impaired accuracy in both memory conditions, producing a decline of $0.59 d^{\prime}$ units in the binding-memory task and a decline of 0.50 $d^{\prime}$ units in the 2-feature memory task (averaged across set sizes). Consistent with these data, an ANOVA with the factors of memory condition (binding or 2-feature), dual-task load (with or without search), and memory set size (three or four items) revealed significant main effects of memory condition, $F(1,48)=10.36, p=$ .002 , and dual-task load, $F(1,48)=48.28, p<.001$. Additionally, although change-detection performance was somewhat better with a set size of three versus four items ( $d^{\prime}=1.30$ vs. 1.12 , respectively), neither the main effect of memory set size nor the DualTask Load $\times$ Memory Set Size interaction approached significance (all $p \mathrm{~s}>.15$ ). Critically, both the Memory Condition $\times$ Dual-Task Load interaction and the Memory Condition $\times$ DualTask Load $\times$ Memory Set Size interaction also failed to reach significance $(F \mathrm{~s}<1)$. Thus, we found no evidence that the search task impaired change detection more in the binding condition than in the 2-feature memory condition.
As an additional test, we compared dual-task change-detection performance in the binding condition with performance in the more difficult feature condition from the 2-feature task. This comparison required us to calculate $d^{\prime}$ separately for each of the feature conditions in the 2 -feature task. To do this, we used the false alarm rate across all of the same trials in the 2-feature condition and the hit rate on different trials calculated separately for each feature condition. ${ }^{3}$ For the dual-task condition (collapsed across set size), binding change-detection performance $\left(d^{\prime}=0.80\right)$ was better than performance for the more difficult feature in the 2-feature condition (orientation; $d^{\prime}=0.50$ ), $F(1,24)=12.93, p<$ .002. Thus, although performance in the binding condition was worse overall than performance in the 2 -feature condition, binding was neither selectively impaired by the search task nor any worse than performance for the more difficult feature in the 2-feature condition, suggesting that binding in VSTM is not differentially dependent on sustained attention. Possible causes for the small but reliable overall decrements in binding found here are considered in the General Discussion.

\footnotetext{
${ }^{3}$ To derive accurate estimates of performance in the two-alternative forced choice design used in change-detection experiments, performance on both same and different trials need to be taken into consideration. That is, the hit rate and the false alarm rate must be considered together. However, because participants are required to monitor two different features on each trial in the 2-feature condition, there is no ideal method for calculating separate false alarm rates for each of the features. Because of this, the exact $d^{\prime}$ values reported separately for each feature in the 2-feature conditions of the present experiments should be taken as estimates only. As a converging measure, for each experiment we also compared the percentage of correct hits in the more difficult of the 2-feature conditions (i.e., orientation) with the percentage of correct hits in the binding condition. In each case, the results of these analyses were identical to the analyses reported in the text, and thus they are not discussed further.
} 
The data from Experiment 1 are inconsistent with the hypothesis that attention plays a special role in the maintenance of feature bindings in VSTM. Although the dual-task manipulation did impair accuracy, it did so equally for binding memory and feature memory, contrary to the predictions of the attention maintenance hypothesis.

However, even though the powerful attention manipulation used in these experiments would seem to have provided every opportunity to find a significant effect of attention on binding memory had there been one, caution must be exercised in drawing conclusions on the basis of a lack of statistical significance. For example, it is possible that attention does indeed play a critical role in binding memory but that we simply lacked the statistical power to detect this effect. Thus, to increase our ability to conclude that withdrawing attention does not differentially impact binding memory, we conducted a confidence interval analysis on the $d^{\prime}$ and percentage correct data from Experiment 1. Specifically, for each participant we computed the difference in accuracy between the 2-feature and binding conditions both with and without the interposed search task. This analysis revealed that the mean difference between the 2 -feature and binding conditions with and without search was $0.09 d^{\prime}$ units, with a $95 \%$ confidence interval of \pm 0.23 $d^{\prime}$ units (this corresponds to a difference of $2 \%$ correct $\pm 3.7 \%$ correct). Thus, we can be $95 \%$ certain that the effect of the search task was between $-0.15(-1.7 \%)$ and $0.32(5.7 \%) d^{\prime}$ units, with any advantage for feature memory over binding memory not greater than $0.32 d^{\prime}$ units, or $5.7 \% .^{4}$

The results from Experiment 1 suggest that the withdrawal of attention does not impair binding memory any more than it impairs feature memory, contrary to the predictions of the attention maintenance hypothesis. These findings suggest that although attention may indeed contribute to the maintenance of VSTM representations in general, it does not play a special role in the maintenance of feature bindings.

\section{Experiment 2A}

In Experiment 1, there was no evidence of a binding-specific deficit when an attentionally demanding task was performed during VSTM maintenance. In Experiments 2-4, we revisited the basic Wheeler and Treisman (2002) effect, which is widely cited as evidence for a binding deficit. However, the results of that study were ambiguous for the reasons discussed previously. To clarify this issue, we conducted Experiments 2-3 to examine whether a whole-array probe at test does indeed produce a specific deficit in memory for feature bindings but not for individual features.

Experiment $2 \mathrm{~A}$ examined this issue by using the stimuli and procedures of Experiment 1, replacing the dual-task manipulation with the manipulation used by Wheeler and Treisman (2002), in which participants were tested with either a whole-array probe or a single-item probe.

\section{Method}

Participants. Twenty University of Iowa undergraduate volunteers ( 9 men and 11 women) participated in the present experiment.

Stimuli. The memory stimuli were identical to those used in Experiment 1, with the exception that no search array appeared during the delay interval, and there were always four memory items.

Design and procedure. Each trial began with the appearance of a small white fixation cross for $500 \mathrm{~ms}$, followed by a 500-ms presentation of the memory display. The memory display was followed by a 1,000-ms delay interval and the appearance of a test display that remained on the screen until the participant made a response. This was followed by a 1,000-ms intertrial interval.

Participants completed the 2-feature memory and binding memory tasks (see the Method section of Experiment 1) with both a single item and a whole display at test. That is, at test, either all four items were presented again at the same locations that were occupied by the memory display items (whole-array test), or a single item was presented at one of the four previously occupied locations (single-item test). In contrast to Experiment $4 \mathrm{~A}$ of Wheeler and Treisman (2002), item position in both conditions was held constant from memory display to test. In all cases, participants indicated whether the item(s) present were the same as or different from the initial display item(s) by pressing one of two buttons on a game pad, with a left-hand response for same and a right-hand response for different. Four blocks of 60 trials each were presented to each participant in a counterbalanced order, for a total of 240 trials. Participants completed 12-16 practice trials before each trial block and received a short break following every 20 trials.

\section{Results and Discussion}

As shown in Figure 3A, 2-feature memory $\left(d^{\prime}=1.73\right)$ was once again more accurate than binding memory $\left(d^{\prime}=1.47\right)$ overall. Additionally, for both memory tasks, accuracy was higher in the whole-array test condition $\left(d^{\prime}=1.93\right)$ than in the single-item test condition $\left(d^{\prime}=1.26\right)$, which is similar to the whole-array advantage observed by Jiang et al. (2000). Consistent with these data, a repeated measures ANOVA with factors of memory condition (2-feature vs. binding) and test-display type (whole array vs. single item) revealed significant main effects of memory condition, $F(1$, $19)=7.51, p<.02$, and test-display type, $F(1,19)=30.35, p<$ .001. Critically, the Memory Condition $\times$ Test-Display Type interaction did not approach significance $(F<1)$, suggesting that 2-feature memory and binding memory were equally impaired when a single item was present at test, in contrast to the findings of Wheeler and Treisman (2002). It should be noted that accuracy in each of the whole-array test conditions was slightly higher than accuracy in the whole-array test conditions of Wheeler and Treisman's Experiment 4A, possibly because Wheeler and Treisman

\footnotetext{
${ }^{4}$ To rule out floor effects as an alternative explanation for a failure to find a significant Memory Condition $\times$ Dual-Task Load interaction, we also repeated our statistical tests, restricting our analysis to the 12 participants in each set-size condition who showed the highest combined accuracy in the 2-feature and binding memory conditions. If the failure to find a significant difference between the two memory conditions in this experiment was due to a floor effect, or to low overall performance, we should see a different pattern of results when analysis is restricted to the high performers. Specifically, the interaction between memory condition and dual-task load should be significant for these participants. Contrary to this proposal, the results for high performers were identical to the results reported for the whole sample.
} 
A

\section{Experiment 2A}

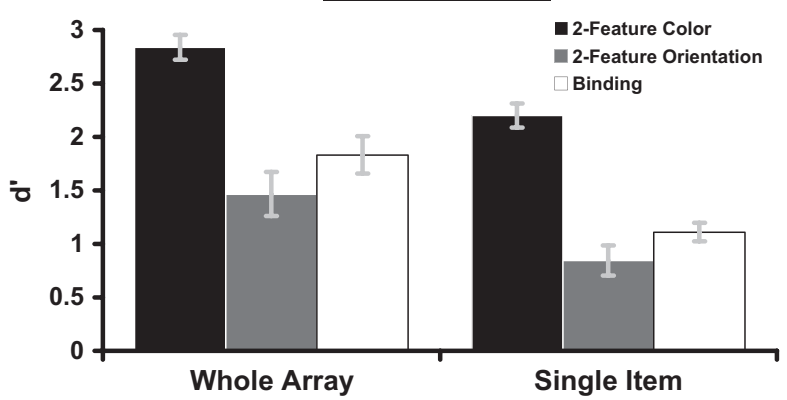

B

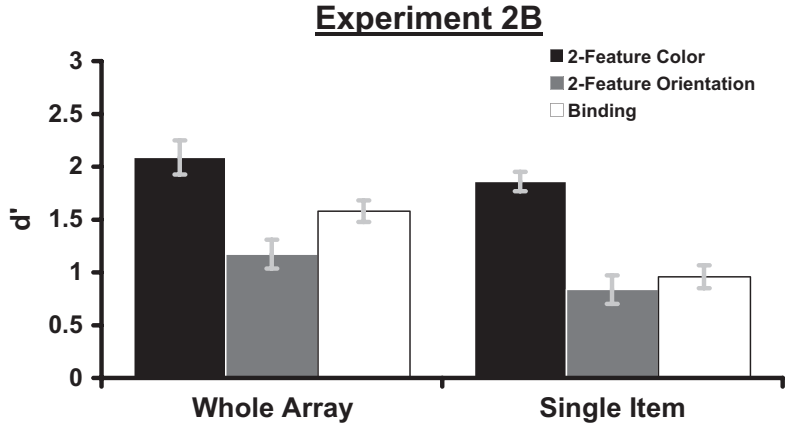

Figure 3. Mean $d^{\prime}$ with either a whole array or a single item at test for the 2-feature and binding memory conditions of Experiment 2A, in which item position was held constant at test (A), and Experiment 2B, in which item position was scrambled at test (B). Error bars reflect 95\% confidence intervals, as described by Loftus and Masson (1994).

scrambled the locations of the objects in the test array, whereas we did not.

Finally, for the whole-array test condition, accuracy for binding change detection $\left(d^{\prime}=1.83\right)$ was again better than accuracy for the more difficult feature in the 2-feature condition (orientation; $d^{\prime}=$ $1.47), F(1,19)=5.65, p<.03$. Binding accuracy $\left(d^{\prime}=1.83\right)$ was slightly less accurate than overall 2-feature accuracy $\left(d^{\prime}=2.04\right)$, but this difference was not significant $(p>.15)$. Thus, both ways of comparing binding accuracy with 2-feature accuracy yielded little or no evidence of a binding deficit in the whole-array test condition.

The data from Experiment 2A help clarify whether a wholearray test generates a binding deficit. No binding deficit was observed, suggesting that the results of Wheeler and Treisman (2002, Experiment 4A) were unlikely to have been caused by the loss of bound object representations when the full array was presented at test.

However, there is one critical difference between the Wheeler and Treisman (2002) method and that of Experiment 2A. Wheeler and Treisman scrambled the locations of objects at test, whereas the objects in Experiment 2A retained their original positions at test. Because of the scrambling, an exhaustive comparison of the sample and test items would require comparison of each of the test array items with each of the items being held in VSTM. If the process of comparing a perceptual representation with a VSTM representation damages or overwrites the VSTM representation, the need to make multiple comparisons could potentially produce a binding deficit. We examined this possibility in Experiment 2B.

\section{Experiment 2B}

In Experiments 1 and 2A, we found no evidence of a bindingspecific deficit in change detection when the locations of items remained consistent from memory display to test. In Experiment $2 \mathrm{~B}$, the positions of objects were scrambled at test. Otherwise, Experiment 2B was the same as Experiment 2A. If this manipulation does in fact lead to difficulties in the comparison process that differentially impact binding, we should see selective deficits in the whole-array binding condition in the present experiment.

The use of scrambled locations in Experiment 2B also made it possible to test an alternative explanation for the lack of a binding deficit in the experiments reported thus far. Recall that binding memory in Experiments 1 and 2A was actually slightly better than memory for the more difficult feature from the 2-feature condition. What could have led to this pattern of results? One possibility is that participants were able to rely on memory for feature-location bindings in addition to feature-feature bindings to generate the same/different responses in the binding condition. Specifically, because location was held constant at test, a correct different response could have been made simply by noticing that one of the remembered colors had changed its location at test, even if the orientation associated with that color had been forgotten. Scrambling locations at test makes it impossible to use this strategy in the binding condition. Thus, if accuracy in the binding condition still remains equal to or better than performance in the more difficult feature condition, we can rule this out as a plausible alternative explanation for preserved binding in Experiments 1 and 2A.

\section{Method}

Participants. Twenty University of Iowa undergraduate volunteers (11 men and 9 women) participated in this experiment.

Procedure. Participants completed the binding and 2-feature memory tasks with either a single item or a whole array at test. The stimuli and procedures were identical to those of Experiment 2A, with the exception that item positions were changed at test. In the single-item test condition, the test item was presented at the center of the screen rather than at one of the four previously occupied locations, whereas in the whole-array test condition, each item was randomly assigned (without replacement) to a different location than it occupied in the initial memory display (see Figure 1A). That is, items in the whole-array test always switched locations from sample to test, regardless of whether a change occurred. These were the procedures used by Wheeler and Treisman (2002).

\section{Results and Discussion}

The results are summarized in Figure 3B. Contrary to the findings of Wheeler and Treisman (2002), accuracy was once again lower in the single-item test condition $\left(d^{\prime}=1.10\right)$ than in the whole-array test condition $\left(d^{\prime}=1.57\right)$. As in Experiment 1 , a repeated measures ANOVA with memory condition (2-feature vs. binding) and test display (single item or whole array) as factors revealed a significant main effect of test display on changedetection performance, $F(1,19)=18.90, p<.001$. However, both the main effect of memory condition and the Memory Condition $\times$ Test Display interaction failed to reach significance $(p s>.14)$.

For the whole-array test condition, accuracy in the binding condition $\left(d^{\prime}=1.58\right)$ was once again higher than accuracy for the 
more difficult feature in the 2 -feature condition (orientation; $d^{\prime}=$ $1.17), F(1,19)=5.01, p<.04$. Binding accuracy $\left(d^{\prime}=1.58\right)$ was nearly identical to overall 2-feature accuracy $\left(d^{\prime}=1.56 ; F<1\right)$. As in Experiment 2A, both ways of comparing binding accuracy with 2-feature accuracy yielded no evidence of a binding deficit in the whole-array test condition. Moreover, it appears that reliance on feature-location binding cannot explain the preserved binding found in Experiments 1 and 2A.

Even with position scrambling in Experiment 2B, there was no evidence of a binding deficit in the whole-array condition, consistent with the results of Experiment 2A.

\section{Experiment 3}

As a final test of whether whole-array probes generate a binding deficit, we conducted a fourth experiment, in which we replicated the methods of Wheeler and Treisman (2002) as exactly as possible. The main change was that we used color and shape rather than color and orientation as the two feature dimensions (with the same shapes used by Wheeler \& Treisman, 2002).

\section{Method}

Participants. Twenty-four University of Iowa undergraduate volunteers (10 men and 14 women) participated in this experiment.

Stimuli. Stimulus presentation and response recording were as described above, with the exception that this experiment was programmed in Matlab 5.2.0 using the extensions provided by Psychophysics Toolbox (Brainard, 1997; Pelli, 1997). Memory stimuli and probe items consisted of small, colored shapes subtending a visual angle of approximately $0.75^{\circ}$ at a viewing distance of $57 \mathrm{~cm}$. The shape of each stimulus was chosen from the following set of eight simple shapes: spiral, hexagram, triangle, hourglass, circle, square, plus sign, and horseshoe (see comparable stimuli in Figure 7 of Wheeler \& Treisman, 2002). The color of each shape was selected from the following set of eight highly discriminable colors: red $\left(x=.65, y=.32 ; 13.65 \mathrm{~cd} / \mathrm{m}^{2}\right)$, yellow $\left(x=.43, y=.48 ; 57.43 \mathrm{~cd} / \mathrm{m}^{2}\right)$, green $(x=.30, y=.58 ; 43.68$ $\left.\mathrm{cd} / \mathrm{m}^{2}\right)$, blue $\left(x=.15, y=.06 ; 7.18 \mathrm{~cd} / \mathrm{m}^{2}\right)$, violet $(x=.29, y=$ $\left..17 ; 25.41 \mathrm{~cd} / \mathrm{m}^{2}\right)$, white $\left(x=.29, y=.28 ; 64.11 \mathrm{~cd} / \mathrm{m}^{2}\right)$, brown $\left(x=.60, y=.31 ; 4.87 \mathrm{~cd} / \mathrm{m}^{2}\right)$, and black $(x=.00, y=.00 ; 0.00$ $\mathrm{cd} / \mathrm{m}^{2}$ ). Individual colors and shapes were never repeated in the initial memory display or at test.

Sample arrays consisted of two, four, or six items presented within an $8.8^{\circ} \times 8.8^{\circ}$ area centered at fixation, with a minimum center-to-center separation between items, and between each item and the center of the screen, of $1^{\circ}$ of visual angle.

Design and procedure. Each trial began with the appearance of a random 3-digit number (for $500 \mathrm{~ms}$ ) that the participant was required to repeat aloud throughout the duration of the trial. After a 500-ms blank interval, the memory display was presented for 150 ms. This was followed by a 900-ms delay and then the appearance of the test array, which remained on the screen until a response was generated. In the single-item test condition, the test item was presented at the center of the screen rather than at one of the previously occupied locations. In the whole-array test condition, each item was randomly assigned (without replacement) to a different location from the one it occupied in the initial memory display, as in Experiment 2B (see Figure 1A). Participants' responses were followed by a 500-ms intertrial interval.
Each participant completed four different memory conditions: a color-only condition, in which the colors of two items changed to new values at test; a shape-only condition, in which the shapes of two items changed to new values at test; a 2-feature condition, in which either the colors or the shapes of two items changed to new values at test; and a binding condition, in which the particular pairing of color and shape changed for two items at test. These four conditions were blocked, with set size balanced across all levels and randomly intermixed within each block. Trial blocks were counterbalanced across participants using a balanced Latin square design. There were 108 experimental trials in each block, for a total of 432 trials. Additionally, participants were given 24 trials of practice before each experimental block, for a total of 96 practice trials.

It should be noted that the procedures in the single-item probe condition reported here differed somewhat from the color/shape single-item probe experiment reported by Wheeler and Treisman (2002, Experiment 4B). Specifically, for color-only and shapeonly trials in their experiment, the test item always had a neutral shape (a square) in the color-only condition and a neutral color (black) in the shape-only condition. As a result, there was always a change in the task-irrelevant dimension. In the present experiment, we elected to keep the stimulus parameters constant across each experimental condition, varying only the nature of the change participants were required to detect at test. That is, the value of the irrelevant dimension was preserved between the sample and test stimuli.

\section{Results and Discussion}

The results are shown in Figure 4. Consistent with the results of Wheeler and Treisman (2002, Experiment 4A), overall accuracy with a whole display at test was highest in the color-only condition $\left(d^{\prime}=2.61\right)$, and worst in the binding condition $\left(d^{\prime}=1.34\right)$. However, in contrast to the findings of Wheeler and Treisman, accuracy in the 2-feature condition $\left(d^{\prime}=1.86\right)$ fell in between the color-only and shape-only $\left(d^{\prime}=1.57\right)$ conditions, in keeping with previous findings (e.g., Luck \& Vogel, 1997). Performance with a single-item probe at test followed a similar pattern, with higher accuracy in the color-only condition $\left(d^{\prime}=2.22\right)$, followed by the 2-feature condition $\left(d^{\prime}=1.59\right)$, the shape-only condition $\left(d^{\prime}=\right.$ $1.48)$, and the binding condition $\left(d^{\prime}=1.30\right)$, in which performance was nearly identical to that observed in the whole-arrray test condition. As with each of our previous experiments, accuracy was once again worse when a single-item probe was presented at test than it was in the whole-array test condition, in keeping with previous findings (Hollingworth, 2005; Jiang et al., 2000).

Consistent with these data, a three-way ANOVA with memory condition (color only, shape only, 2-feature, binding) and set size (two, four, or six items) as within-subject factors and test display type (whole array, single-item probe) as a between-subjects factor revealed significant main effects of memory condition, $F(3,66)=$ 27.68, $p<.001$, and set size, $F(2,44)=216.19, p<.001$. However, neither the main effect of test display type nor any of the interactions involving test display type approached significance $(p s>.20)$, except that the Memory Condition $\times$ Set Size interaction was marginally significant, $F(6,132)=2.00, p=.07$.

To unpack the main effect of memory condition, we also ran a series of paired-samples $t$ tests comparing accuracy between each 


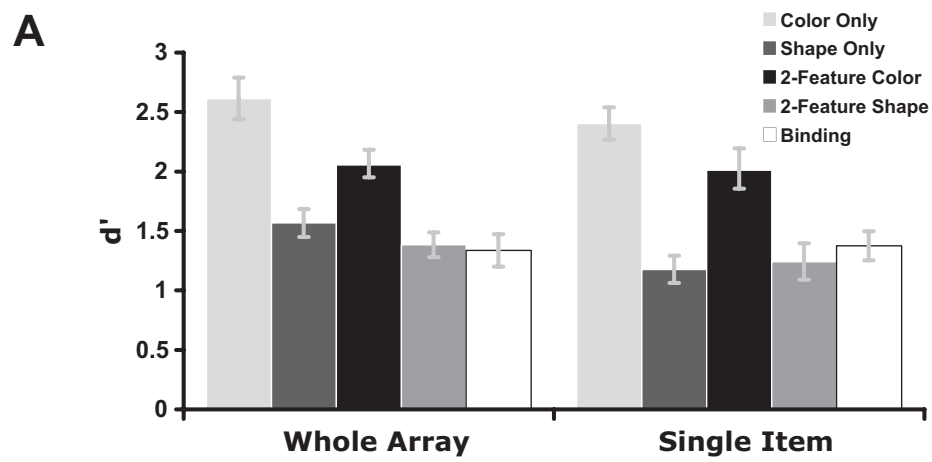

B

Whole Array

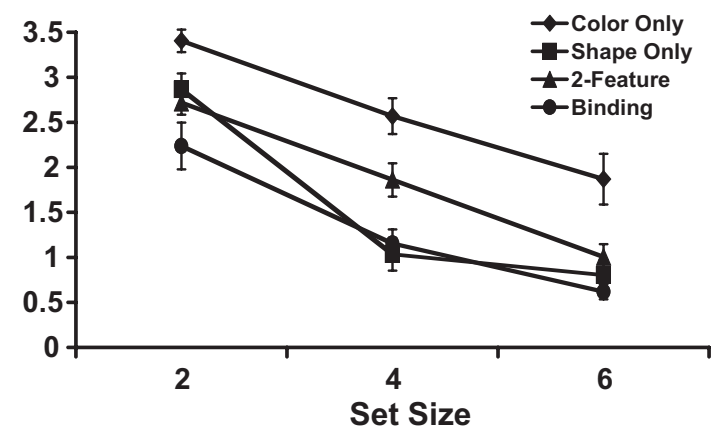

Single Item

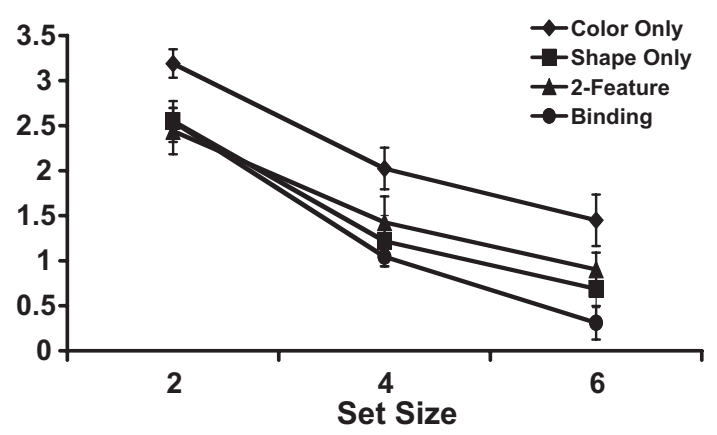

Figure 4. Mean $d^{\prime}$ data from Experiment 3, collapsed across set size (A) or broken down by set size (B). Error bars reflect $95 \%$ confidence intervals, as described by Loftus and Masson (1994).

of the different memory conditions with both a single-item probe and a whole display at test. These analyses used a Bonferroni correction for multiple comparisons; consequently, an uncorrected $p$ value of $<.008$ is necessary to achieve a corrected $p$ value of $<$ .05 . In the single-item probe analyses, overall accuracy in the color-only condition was indeed significantly higher than accuracy in each of the other three conditions, $t \mathrm{~s}(11) \geq 4.21$, uncorrected $p s<.006$, whereas the other three conditions were not significantly different from each other when corrected for multiple comparisons (uncorrected $p s>.03$ ). Similarly, the whole-array test analyses revealed that accuracy was significantly better in the color-only condition than in each of the other conditions, $t \mathrm{~s}(11) \geq$ 4.92, uncorrected $p \mathrm{~s}<.001$. Accuracy was significantly worse in the binding condition than in the 2 -feature condition, $t(11)=3.39$, uncorrected $p<.007$. However, accuracy in the binding condition was not significantly worse than accuracy in the shape-only condition, and the shape-only and 2-feature conditions did not significantly differ from each other (uncorrected $p s>.02$ ).

Finally, for the whole-array test condition, accuracy for the binding condition $\left(d^{\prime}=1.34\right)$ was nearly identical to accuracy for the more difficult feature in the 2-feature condition (shape; $d^{\prime}=$ 1.38; $F<1)$. Thus, although performance in the whole-array binding condition $\left(d^{\prime}=1.34\right)$ was worse overall than performance in the 2-feature condition $\left(d^{\prime}=1.86\right)$, binding was no worse than performance for the more difficult feature in the 2-feature condition or than performance in the single-feature shape condition $\left(d^{\prime}\right.$ $=1.57$ ). Moreover, performance in the binding condition was nearly identical across the single-item probe and whole-array test conditions, suggesting that binding was not selectively impaired by the appearance of the whole array at test.

As in Experiments 2A and 2B, the results of the present experiment showed no consistent evidence that binding performance was more impaired in the whole-array test condition than in the single-item probe condition. Although accuracy tended to be lower for the binding condition than for the 2-feature condition, this difference was observed for both the whole-array and single-item test conditions, and the interaction between test type and memory condition did not approach significance. Moreover, accuracy for the binding condition was approximately the same as accuracy for the more difficult of the two individual features. Together, these experiments provide consistent evidence against the hypothesis that the appearance of multiple items at test results in a selective disruption of binding memory. In addition, the findings reported here are generally consistent with other findings in this area (e.g., Hollingworth, 2005; Jiang et al., 2000; Luck \& Vogel, 1997), in which performance has been consistently better for whole-array probes than for single-item probes. This argues against any type of attention-induced deficit in the whole-array probe condition.

\section{General Discussion}

In this study, we investigated the role of attention in the maintenance of bound object representations in VSTM. Attention has been found to play an important role in binding features into perceptual object representations (Treisman \& Gelade, 1980). In addition, attention supports the transfer of perceptual information 
into VSTM (Averbach \& Coriell, 1961; Hollingworth \& Henderson, 2002; Irwin, 1992; Schmidt, Vogel, Woodman, \& Luck, 2002; Sperling, 1960).

Is attention also required to maintain feature bindings in VSTM? The literature to date has provided conflicting answers to this question. On the one hand, Rensink (2000), Wheeler and Treisman (2002), and others (e.g., Wolfe, 1999) have answered in the affirmative, suggesting that bound object representations collapse into unbound collections of features following the withdrawal of attention. On the other hand, Luck and Vogel (1997; Vogel et al., 2001), Hollingworth and colleagues (Hollingworth, 2003; Hollingworth \& Henderson, 2002; Hollingworth et al., 2001), and others (Irwin, 1992; Irwin \& Zelinsky, 2002) have suggested that a limited number of bound object representations can be maintained in VSTM in the absence of focused attention.

Wheeler and Treisman (2002) based their conclusion on the finding of binding-specific deficits when all of the sample items were presented at test (i.e., the whole-array test condition). They explained these results by proposing that attention is required to maintain feature bindings, but not individual features, in VSTM. The presence of distractors in the whole-array condition caused attentional distraction, leading to disruption of memory for feature bindings. However, Wheeler and Treisman did not directly manipulate attention, limiting their ability to isolate attention as the critical factor in the observed binding deficit. In addition, the assumption that whole-array presentation at test leads to attentional distraction and change-detection decrements is undermined by the finding that change detection is generally better under whole-array test conditions than under single-probe test conditions (Hollingworth, 2005; Jiang et al., 2000). Moreover, the binding decrements observed by Wheeler and Treisman were relatively small, and binding memory in the whole-array conditions remained well above chance. Even if we assume that the whole-array test led to attentional distraction, this distraction did not greatly disrupt binding memory.

In the present study, we found no evidence of a whole-array binding deficit, even when we closely followed the original Wheeler and Treisman (2002) paradigm. Instead, we consistently found superior memory performance in the whole-array conditions compared with the single-probe conditions, demonstrating that whole-array presentation does not necessarily lead to distraction and memory performance decrements. It is not clear why we were unable to replicate Wheeler and Treisman's finding of a binding decrement under whole-array test but not single-item test conditions. As discussed in the introduction, the color-shape binding decrement observed by Wheeler and Treisman was small, and it was not clear whether this decrement was statistically significant. That we have failed to find this effect in several attempts and under a range of conditions suggests that this effect is not robust and general.

More important, however, we directly manipulated attention in Experiment 1 by introducing a demanding visual search task during the retention interval of the change-detection task, making it possible to directly test the hypothesis that attention plays a role in the maintenance of bindings in VSTM. The specific visual search task used in Experiment 1 provided a particularly potent manipulation of attention, requiring serial shifts of attention to search objects and perceptual processing of each attended search object (Woodman \& Luck, 1999, 2003). Therefore, if the mainte- nance of bindings, but not features, in VSTM depends on the sustained allocation of attention, only binding memory should have been substantially impaired when the search task was performed. Contrary to this prediction, we found that binding memory and feature memory were equally impaired when the search task was performed during the delay interval. This suggests that although an attention-demanding secondary task can impair VSTM for object properties and their bindings, attention is not playing a special role in the maintenance of the feature bindings themselves (see also Allen et al., 2006; Gajewski \& Brockmole, 2006).

Although attention does not appear to be uniquely required to maintain feature bindings in VSTM, it might be tempting to conclude that attention does play a role in the maintenance of information in VSTM more generally, one similar to its proposed role in the maintenance of information in spatial working memory (Awh \& Jonides, 1998). However, drawing such a conclusion on the basis of the present experiments would be premature. This is because, in addition to being a potent manipulation of perceptuallevel attention, the search task used in Experiment 1 also involves multiple other processes, including the representation of the search target in memory, the perceptual analysis of each of the attended display items, the comparison of each item with a representation of the search target, and the generation of the decision and response required by the task. As a result, the dual-task performance decrements found in our experiments may not have been caused by the spatial-attention demands of the search task. For example, previous studies have shown that focusing attention on a spatial location, either voluntarily or involuntarily, increases the likelihood that the information at that location will be consolidated in VSTM (Duncan \& Humphreys, 1989; Irwin \& Gordon, 1998; Schmidt et al., 2002). Thus, the change-detection decrements seen in the dual-task conditions of Experiment 1 could have been caused by the disruption or replacement of information in VSTM as attention was sequentially focused on the locations of the items making up the search array rather than by the withdrawal of attention per se.

Nonetheless, the findings reported here do provide additional support for the idea that attention is needed throughout the various stages of the change-detection task. Previous research has shown that both the encoding of information in VSTM and the comparison of items in memory with the test array are capacity-limited processes (Hyun, 2006; Vogel, Woodman, \& Luck, in press). Additionally, several other studies have demonstrated that VSTM is impaired when a secondary task is completed during the delay interval (Allen et al., 2006; Fougnie \& Marois, 2006; Makovski, Shim, \& Jiang, in press). This suggests that attention may play a much more general role in change detection than the role suggested by Wheeler and Treisman (2002).

Another issue that needs to be addressed is the finding of general binding decrements in our experiments. That is, although binding was not differentially impacted by the secondary load task or by the nature of the test display in our experiments, there were small but consistent (if not always statistically significant) overall decrements in performance in the binding conditions reported here. What can account for impaired binding in the present study? One possibility is that global statistical properties can be remembered in addition to specific information about individual items, which would aid in the detection of new features but would not aid in the detection of binding changes. For example, observers may be able to remember the average color or average orientation of the sample 
array, and the presence of a new feature value in the test array would lead to a different average color or average orientation. The detection of changes in the average values could produce a small improvement in performance in conditions that involve changing the feature values between the sample and test arrays. However, the average color and average orientation do not change in the binding condition, so this condition would not be aided by the use of memory for statistical properties. Thus, the use of memory for statistical properties of the images would lead to better performance for feature-change conditions than for binding-memory conditions.

In keeping with this possibility, several studies have demonstrated that participants are quite capable of computing statistical descriptors of stimulus arrays. For example, Chong and Treisman (2003, 2005) have demonstrated that judgments of the mean sizes of the items in an array are almost as accurate as size judgments for single stimuli and that such judgments rely on an automatic and parallel computation of mean size by the visual system. Similarly, other studies have revealed an impressive ability of the visual system to use statistical properties in judgments of the direction and speed of motion (Watamaniuk \& Duchon, 1992; Williams \& Sekuler, 1984) and the orientation (Dakin, 1997; Dakin \& Watt, 1997) of the items in multielement displays.

Finally, despite our finding of slightly worse performance for bindings than for features, we found that memory for bindings was preserved quite well despite the attentional demands of the search task. The search task used in Experiment 1 is known to require serial shifts of attention to individual objects within the search array (Woodman \& Luck, 1999, 2003). Thus, with a set size of eight items, attention was likely to have been shifted to four different objects, on average, before the target was identified. This suggests a rather slow search rate of around 200-300 ms per item in the binding memory condition (mean RT $=1,180 \mathrm{~ms}$ ), making this a very difficult search task. Moreover, because pairs of items in the search array were placed in separate quadrants centered more than $5^{\circ}$ away from fixation, participants almost certainly made one or more saccadic eye movements before finding the target. Attention has been shown to be obligatorily and exclusively focused on the location of an intended saccade target prior to the actual eye movement (e.g., Hoffman \& Subramaniam, 1995), making it highly unlikely that spatial attention remained focused on the contents of VSTM during the delay. Thus, the use of this search task in Experiment 1 constituted a stringent test of whether bound object information can be preserved after the withdrawal of attention from an object. Despite serial shifts of attention to multiple objects during the retention interval, memory performance remained near $65 \%$ correct in the dual-task binding condition, suggesting that bound object representations can be maintained as attention is shifted from object to object. This could account for the finding of preserved memory for object information following the withdrawal of attention in previous studies (Hollingworth, 2004; Hollingworth \& Henderson, 2002; Irwin \& Zelinsky, 2002; Zelinsky \& Loschky, 2005). Preserved memory for bound object representations following shifts of the eyes and attention may play an important role in the creation of longer term scene representations (Hollingworth, 2004; Hollingworth \& Henderson, 2002).

\section{References}

Allen, R. A., Baddeley, A. D., \& Hitch, G. J. (2006). Is the binding of visual features in working memory resource-demanding? Journal of Experimental Psychology: General, 135, 298-313.

Averbach, E., \& Coriell, A. S. (1961). Short-term memory in vision. Bell System Technical Journal, 40, 309-328.

Awh, E., \& Jonides, J. (1998). Spatial working memory and spatial selective attention. In R. Parasuraman (Ed.), The attentive brain (pp. 71-94). Cambridge, MA: MIT Press.

Baddeley, A. D. (1986). Working memory. Oxford, England: Clarendon Press.

Baddeley, A. D., \& Logie, R. H. (1999). Working memory: The multiplecomponent model. In A. Miyake \& P. Shah (Eds.), Models of working memory (pp. 28-61). Cambridge, England: Cambridge University Press.

Bates, T. C., \& D'Oliveiro, L. (2003). PsyScript: A Macintosh application for scripting experiments. Behavior Research Methods, Instruments, and Computers, 35, 565-576.

Besner, D., Davies, J., \& Daniels, S. (1981). Reading for meaning: The effects of concurrent articulation. Quarterly Journal of Experimental Psychology: Human Experimental Psychology, 33(A), 415-437.

Brainard, D. H. (1997). The Psychophysics Toolbox. Spatial Vision, 10, 433-436.

Chong, S. C., \& Treisman, A. M. (2003). Representation of statistical properties. Vision Research, 43, 393-404.

Chong, S. C., \& Treisman, A. M. (2005). Statistical processing: Computing the average size in perceptual groups. Vision Research, 45, 891-900.

Courtney, S. M., Ungerleider, L. G., Keil, K., \& Haxby, J. V. (1996). Object and spatial visual working memory activate separate neural systems in human cortex. Cerebral Cortex, 6, 39-49.

Cowan, N. (2001). The magical number 4 in short-term memory: A reconsideration of mental storage capacity. Behavioral and Brain Sciences, 24, 87-185.

Dakin, S. C. (1997). The detection of structure in glass patterns: Psychophysics and computational models. Vision Research, 37, 2227-2246.

Dakin, S. C., \& Watt, R. J. (1997). The computation of orientation statistics from visual texture. Vision Research, 37, 3181-3192.

Delvenne, J.-F., Braithwaite, J. J., Riddoch, M. J., \& Humphreys, G. W. (2002). Capacity limits in visual short-term memory for local orientations. Current Psychology of Cognition, 21, 681-690.

Duncan, J., \& Humphreys, G. W. (1989). Visual search and stimulus similarity. Psychological Review, 96, 433-458.

Fougnie, D., \& Marois, R. (2006). Distinct capacity limits for attention and working memory. Psychological Science, 17, 526-534.

Gajewski, D. A., \& Brockmole, J. R. (2006). Feature bindings endure without attention: Evidence from an explicit recall task. Psychonomic Bulletin \& Review, 13, 581-587.

Hoffman, J. E., \& Subramaniam, B. (1995). The role of visual attention in saccadic eye movements. Perception \& Psychophysics, 57, 787-795.

Hollingworth, A. (2003). Failures of retrieval and comparison constrain change detection in natural scenes. Journal of Experimental Psychology: Human Perception and Performance, 29, 388-403.

Hollingworth, A. (2004). Constructing visual representations of natural scenes: The roles of short- and long-term visual memory. Journal of Experimental Psychology: Human Perception and Performance, 30, 519-537.

Hollingworth, A. (2005). The relationship between online visual representation of a scene and long-term scene memory. Journal of Experimental Psychology: Learning, Memory, and Cognition, 31, 396-411.

Hollingworth, A., \& Henderson, J. M. (2002). Accurate visual memory for previously attended objects in natural scenes. Journal of Experimental Psychology: Human Perception and Performance, 28, 113-136.

Hollingworth, A., Williams, C. C., \& Henderson, J. M. (2001). To see and remember: Visually specific information is retained in memory from 
previously attended objects in natural scenes. Psychonomic Bulletin \& Review, 8, 761-768.

Horowitz, T. S., \& Wolfe, J. M. (1998, August 6). Visual search has no memory. Nature, 394, 575-577.

Hyun, J.-S. (2006). How are visual working memory representations compared with perceptual inputs? Unpublished manuscript, University of Iowa.

Irwin, D. E. (1992). Memory for position and identity across eye movements. Journal of Experimental Psychology: Learning, Memory, and Cognition, 18, 307-317.

Irwin, D. E., \& Gordon, R. D. (1998). Eye movements, attention and trans-saccadic memory. Visual Cognition, 5, 127-155.

Irwin, D. E., \& Zelinsky, G. J. (2002). Eye movements and scene perception: Memory for things observed. Perception \& Psychophysics, 64, 882-895.

Jiang, Y., Olson, I. R., \& Chun, M. M. (2000). Organization of visual short-term memory. Journal of Experimental Psychology: Learning, Memory, and Cognition, 26, 683-702.

Kahneman, D., Treisman, A. M., \& Gibbs, B. J. (1992). The reviewing of object files: Object-specific integration of information. Cognitive Psychology, 24, 175-219.

Loftus, G. R., \& Masson, M. E. J. (1994). Using confidence intervals in within-subjects designs. Psychonomic Bulletin \& Review, 1, 476-490.

Luck, S. J., \& Beach, N. J. (1998). Visual attention and the binding problem: A neurophysiological perspective. In R. D. Wright (Ed.), Visual attention (pp. 455-478). New York: Oxford University Press.

Luck, S. J., \& Vogel, E. K. (1997, November 20). The capacity of visual working memory for features and conjunctions. Nature, 390, 279-281.

Makovski, T., Shim, W.-M., \& Jiang, Y. (in press). Interference from filled delays on visual change detection. Journal of Vision.

Murray, D. J. (1968). Articulation and acoustic confusability in short-term memory. Journal of Experimental Psychology, 78, 679-684.

Olson, I. R., \& Jiang, Y. (2002). Is visual short-term memory object based? Rejection of the "strong-object" hypothesis. Perception \& Psychophysics, 64, 1055-1067.

O'Regan, J. K. (1992). Solving the "real" mysteries of visual perception: The world as an outside memory. Canadian Journal of Psychology, 46, 461-488.

O'Regan, J. K., \& Nöe, A. (2001). A sensorimotor account of vision and visual consciousness. Behavioral and Brain Sciences, 24, 939-1011.

Pashler, H. (1988). Cross-dimensional interaction and texture segregation. Perception \& Psychophysics, 43, 307-318.

Pelli, D. G. (1997). The Video Toolbox software for visual psychophysics: Transforming numbers into movies. Spatial Vision, 10, 437-442.

Phillips, W. A. (1974). On the distinction between sensory storage and short-term visual memory. Perception \& Psychophysics, 16, 283-290.

Rensink, R. A. (2000). The dynamic representation of scenes. Visual Cognition, 7, 17-42.

Rensink, R. A. (2002). Change detection. Annual Review of Psychology, 53, 245-277.

Roskies, A. L. (1999). The binding problem. Neuron, 24, 7-9.

Saiki, J., \& Miyatsuji, H. (in press). Feature binding in visual working memory evaluated by type identification paradigm. Cognition.
Schmidt, B. K., Vogel, E. K., Woodman, G. F., \& Luck, S. J. (2002). Voluntary and automatic attentional control of visual working memory. Perception \& Psychophysics, 64, 754-763.

Simons, D. J. (1996). In sight, out of mind: When object representations fail. Psychological Science, 7, 301-305.

Simons, D. J., \& Levin, D. T. (1997). Change blindness. Trends in Cognitive Sciences, 1, 261-267.

Sperling, G. (1960). The information available in brief visual presentations. Psychological Monographs, 74(Whole No. 498).

Stefurak, D. L., \& Boynton, R. M. (1986). Independence of memory for categorically different colors and shapes. Perception \& Psychophysics, 39, 164-174.

Tootell, R. B. H., Dale, A. M., Sereno, M. I., \& Malach, R. (1998). New images from human visual cortex. Trends in Neuroscience, 19, 481-489.

Treisman, A. M. (1993). The perception of features and objects. In A. D. Baddeley \& L. Weiskrantz (Eds.), Attention: Selection, awareness and control: A Tribute to Donald Broadbent (pp. 5-35). Oxford, England: Clarendon Press.

Treisman, A. M. (1996). The binding problem. Current Opinion in Neurobiology, 6, 171-178.

Treisman, A. M., \& Gelade, G. (1980). A feature-integration theory of attention. Cognitive Psychology, 12, 97-136.

Vogel, E. K., Woodman, G. F., \& Luck, S. J. (2001). Storage of features, conjunctions, and objects in visual working memory. Journal of Experimental Psychology: Human Perception and Performance, 27, 92-114.

Vogel, E. K., Woodman, G. F., \& Luck, S. J. (in press). The time course of consolidation in visual working memory. Journal of Experimental Psychology: Human Perception and Performance.

Watamaniuk, S. N. J., \& Duchon, A. (1992). The human visual system averages speed information. Vision Research, 32, 931-941.

Wheeler, M., \& Treisman, A. M. (2002). Binding in short-term visual memory. Journal of Experimental Psychology: General, 131, 48-64.

Williams, D. W., \& Sekuler, R. (1984). Coherent global motion percepts from stochastic local motions. Vision Research, 24, 55-62.

Wolfe, J. M. (1999). Inattentional amnesia. In V. Coltheart (Ed.), Fleeting memories (pp. 71-94). Cambridge, MA: MIT Press.

Woodman, G. F., \& Luck, S. J. (1999, August 26). Electrophysiological measurement of rapid shifts of attention during visual search. Nature, 400, 867-869.

Woodman, G. F., \& Luck, S. J. (2003). Serial deployment of attention during visual search. Journal of Experimental Psychology: Human Perception and Performance, 29, 121-138.

$\mathrm{Xu}, \mathrm{Y}$. (2002). Limitations of object-based feature encoding in visual short-term memory. Journal of Experimental Psychology: Human Perception and Performance, 28, 458-468.

$\mathrm{Xu}, \mathrm{Y}$. (2004). An object benefit for encoding two color features of an object in visual short term memory. Paper presented at the 45th Annual Meeting of the Psychonomic Society, Minneapolis, MN.

Yeh, Y.-Y., Yang, C.-T., \& Chiu, Y.-C. (2005). Binding or prioritization: The role of selective attention in visual short-term memory. Visual Cognition, 12, 759-799.

Zelinsky, G. J., \& Loschky, L. C. (2005). Eye movements serialize memory for objects in scenes. Perception \& Psychophysics, 67, 676-690. 


\section{Appendix}

Proportions of Hits and False Alarms (FAs) for Experiments 1-3

\begin{tabular}{|c|c|c|c|c|}
\hline \multirow[b]{2}{*}{ Set size and/or search condition } & \multicolumn{4}{|c|}{ Hits/FAs } \\
\hline & Color-only & Shape-only & 2-feature & Binding \\
\hline \multicolumn{5}{|c|}{ Experiment 1} \\
\hline \multicolumn{5}{|l|}{ Set size 3} \\
\hline No search & & & $.76 / .21$ & $.70 / .22$ \\
\hline Search & & & $.70 / .30$ & $.61 / .31$ \\
\hline \multicolumn{5}{|l|}{ Set size 4} \\
\hline No search & & & $.68 / .19$ & $.65 / .19$ \\
\hline Search & & & $.62 / .26$ & $.57 / .30$ \\
\hline \multicolumn{5}{|c|}{ Experiment $2 \mathrm{~A}$} \\
\hline Whole array & & & $.78 / .14$ & $.71 / .14$ \\
\hline Single item & & & $.74 / .25$ & $.70 / .30$ \\
\hline \multicolumn{5}{|c|}{ Experiment $2 \mathrm{~B}$} \\
\hline Whole array & & & $.72 / .20$ & $.72 / .17$ \\
\hline Single item & & & $.81 / .38$ & $.73 / .39$ \\
\hline \multicolumn{5}{|c|}{ Experiment 3} \\
\hline \multicolumn{5}{|l|}{ Set size 2} \\
\hline Whole array & $.98 / .06$ & $.96 / .14$ & $.96 / .18$ & $.81 / .12$ \\
\hline Single item & $.96 / .07$ & $.91 / .14$ & $.86 / .13$ & $.88 / .12$ \\
\hline \multicolumn{5}{|l|}{ Set size 4} \\
\hline Whole array & $.88 / .12$ & $.60 / .25$ & $.77 / .17$ & $.56 / .19$ \\
\hline Single item & $.91 / .29$ & $.75 / .35$ & $.82 / .37$ & $.72 / .34$ \\
\hline \multicolumn{5}{|l|}{ Set size 6} \\
\hline Whole array & $.72 / .13$ & $.45 / .19$ & $.54 / .21$ & $.45 / .24$ \\
\hline Single item & $.80 / .33$ & $.68 / .44$ & $.84 / .56$ & $.73 / .64$ \\
\hline
\end{tabular}

Received January 9, 2006

Revision received March 15, 2007

Accepted April 11, 2007 